\title{
Magnetic and Transport Properties of (III,Mn)V Ferromagnetic Semiconductors
}

\author{
T. Jungwirth ${ }^{a, b}$, J. Sinova ${ }^{b, c}$ And A.H. MacDonald ${ }^{b}$ \\ ${ }^{a}$ Institute of Physics ASCR, Cukrovarnická 10, 16253 Praha 6, Czech Republic \\ ${ }^{b}$ Department of Physics, The University of Texas, Austin, TX 78712, USA \\ ${ }^{c}$ Texas A \& M University, College Station, TX 77843-4242, USA \\ The basic microscopic origins of ferromagnetism in (III,Mn) V com- \\ pounds that have the highest transition temperatures appear to be well \\ understood, and efficient computation methods have been developed which \\ are able to model their magnetic, transport, and optical properties. We re- \\ view some of the attempts over the past five years to achieve a theoretical \\ understanding of these complex magnetic systems.
}

PACS numbers: 71.20.Nr, 75.30.Et, 75.50.Pp

\section{Introduction}

Ferromagnetic semiconductors are unique materials that can realize the full potential of a spintronic device which utilizes a controlled flow of electronic charge and spin. They offer integration of magnetic, semiconducting, and optical properties, and compatibility with existing microelectronic technologies. An important milestone in the field of semiconductor spintronics was the discovery five years ago of ferromagnetism in Mn-doped, $p$-type GaAs observed at temperatures in excess of $100 \mathrm{~K}$ [1]. Ferromagnetism at room temperature with full participation of itinerant carriers would be a major breakthrough in semiconductor spintronics. With this aim, intensive material research is currently in progress on transition metal doped III-V semiconductors. Here we present a brief review of several theoretical works on magnetic, transport, and optical properties of (III,Mn) V ferromagnetic semiconductors, which has progressed hand in hand with experimental developments.

A qualitative picture of the electronic structure of III-V diluted magnetic semiconductors (DMSs) was proposed by Dietl and co-workers [2,3]. Their model is based on the internal reference rule [4] which states that energy levels derived from the $d$-shell of the magnetic ion are constant across semiconductor compound 
families with properly aligned bands. The application of this rule to III-V materials with ionized magnetic impurities substituting on cation sites was discussed in detail in Refs. $[2,3,5,6]$. The position of the $3 d$ level for $\mathrm{Mn}$ in GaAs, deep in the valence band, suggests that $\mathrm{Mn}$ in GaAs is $2+$, that its $d$-shell is occupied by five electrons, and that incorporation of $\mathrm{Mn}$ in this and many other (III,Mn) V compounds will result in a large density of valence band holes that can mediate ferromagnetic coupling between the $S=5 / 2 \mathrm{Mn}$ local moments. For low carrier densities, these valence band holes will be bound to the $\mathrm{Mn}$ ion, leading to shallow acceptor levels. This model of carrier-induced ferromagnetism is now well established for (Ga,Mn)As as a result of electron paramagnetic resonance experiments [7], X-ray magnetic circular dichroism measurements [8, 9], magneto-transport data [10-13], and theory works which we partly discuss in more detail below. If applicable to arsenides, the $\mathrm{Mn}^{2+}$ shallow acceptor picture might even more accurately describe Mn impurity states in the narrow gap InSb and GaSb host compounds. This has recently been confirmed by experimental and theoretical studies of ferromagnetic (In,Mn)Sb DMSs [14].

The acceptor impurity levels of Fe and Co [2-5] are unlikely to lead to high valence band hole concentrations in arsenides or antimonides.

The possible coexistence of acceptor and neutral magnetic impurities suggests that a ferromagnetic double-exchange mechanism can dominate in this case [15]. The scenario, however, has not yet been confirmed experimentally. For nitrides and phosphides the ionization energy of the acceptor levels is even higher and the $3 d \mathrm{Mn}$ impurity level is not deep in the valence band, making it likely that $d$-charge fluctuations cannot be neglected. Experimentally, ferromagnetism with critical temperatures exceeding room temperature has been claimed in ( $\mathrm{Ga}, \mathrm{Mn}) \mathrm{N}$ $[16,17]$ on the basis of hysteresis in magnetization measurements, however, the electronic configuration of Mn impurity in this compound has not yet been conclusively established. The observation of the $p$-type conduction achieved in some samples [18] seems to suggest that the simple picture of Mn acceptor level according to the internal reference rule may not apply in this case.

Guided by the complex phenomenology very briefly discussed above and by available experimental data on III-V DMSs, several theoretical groups have performed detailed studies of these new ferromagnetic materials using both microscopic and effective Hamiltonian approaches. The results of some of this theoretical research are reviewed in the following paragraphs.

\section{Ab initio theories}

$A b$ initio theories are an invaluable tool for studying the microscopic origins of ferromagnetism and for predicting electronic, magnetic, and structural ground-state properties of new compounds. The local density approximation (LDA) combined with disorder-averaging coherent-potential approximation (CPA) was 
used [19] to study GaAs and GaN DMSs doped with V, Cr, Mn, Fe, Co, and Ni. A ferromagnetic ground state was predicted for $\mathrm{V}, \mathrm{Cr}$, and $\mathrm{Mn}$ doping, while $\mathrm{Fe}$, $\mathrm{Co}$, and Ni impurities were predicted to lead to a spin-glass state. The structural properties of $(\mathrm{Ga}, \mathrm{Mn})$ As have been calculated in the dilute limit and for hypothetical zinc-blende MnAs using pseudoatomic-orbital-based LDA algorithms [20]. The calculated lattice constant of MnAs is similar to the lattice constant of GaAs. This result and subsequent calculations for (Ga,Mn)As DMSs [21] suggest that the relatively large compressive strains observed in ( $\mathrm{Ga}, \mathrm{Mn})$ As epilayers grown on GaAs are caused by intrinsic defects rather than by the substitutional Mn.

The electronic structure of (Ga,Mn)As supercells with $\mathrm{Mn}$ in the substitutional position, with As-antisites, or Mn-interstitial defects have been studied by various methods within the LDA $[20,22,23]$. It has been conclusively established by the LDA calculations that in the presence of substitutional Mn, the Fermi energy crosses the valence band near the $\Gamma$ point, introducing one hole per Mn. Clear theoretical evidence for As $4 p$-like band holes has been obtained in self-interaction corrected LDA and LDA+U calculations [24] and the Mn $3 d$ density of states calculated by this method is in good agreement with photoemission data [25]. As-antisite and Mn-interstitial defects act as a double donor, according to the microscopic calculations [20,22, 23], and, therefore, are the likely sources of carrier compensation observed in experiment. In summary, first-principles calculations support the picture of carrier induced ferromagnetism in some (III,Mn) V ferromagnets, but detailed predictions depend on the approximation used for exchange and correlations and also perhaps on technical details of the calculation. This activity is on-going and further progress should be expected.

\section{Semi-phenomenological effective Hamiltonian theories}

Semi-phenomenological effective Hamiltonian theories in which the low-energy degrees of freedom are $\mathrm{Mn}$ ion $S=5 / 2$ spins and valence band holes, discussed in the following paragraphs, have been developed to make quantitative predictions for zero and finite temperature magnetic, transport, and optical properties of bulk samples [2, 26] and heterostructures [27]. In (Ga,Mn)As, e.g., these models are partially justified by the negligible changes in the valence band structure of the host semiconductor observed in angle-resolved photoemission experiments [28]. The key term in the effective Hamiltonian description is a Kondo-like exchange interaction, $J_{p d} \sum_{I} \boldsymbol{S}_{I} \cdot s\left(\boldsymbol{R}_{I}\right)$, which arises microscopically from $s p-d$ hybridization and strong $d$-orbital Coulomb interactions. Here $\boldsymbol{S}_{I}$ is the $S=5 / 2$ local moment operator on Mn site $I$ and $s(r)$ is the multi-band envelope function hole spin-operator $[29,30]$. The simplest version of this model combines mean-field theory with a virtual crystal approximation for the random distribution of Mn moments [2, 31, 26, 32]. Illustrative results of the model are presented in Fig. 1 for the critical temperature in $(\mathrm{Ga}, \mathrm{Mn}) \mathrm{As}$ and $(\mathrm{In}, \mathrm{Mn})$ As. These calculations and the model predictions for 


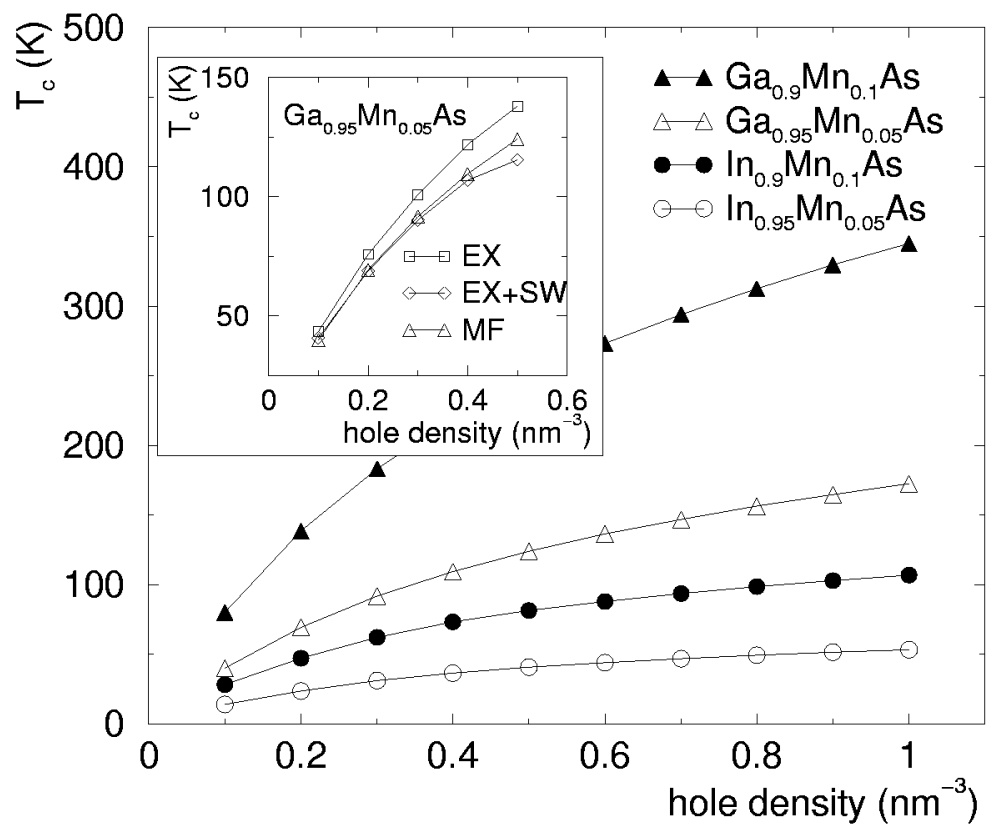

Fig. 1. Main plot: mean-field critical temperature calculated using the effective Hamiltonian and virtual crystal approximations for (Ga,Mn)As (triangles) and (In, Mn)As (circles) DMSs as a function of the hole density [29, 37]. Inset: critical temperatures in (Ga,Mn)As, obtained by including hole-hole exchange interaction (EX) and spin-wave fluctuations $(\mathrm{EX}+\mathrm{SW})$, are compared to the mean-field results (MF).

the strain-engineered magnetic anisotropy [29, 30], anomalous Hall conductivity [33, 34], domain structure [35], and magnetic circular dichroism [29] are in good agreement with experiment.

The reliability of the standard mean-field approximation has been examined by accounting for the Coulomb interactions among holes [26], which enhance the critical temperature, and for correlations in Mn moment orientations which reduce the energetic cost of incompletely aligned spin configurations [36] and therefore lower the critical temperature. The calculations show that heavy-hole-light-hole mixing is responsible for a relatively large spin stiffness, resulting in only $\approx 20 \%$ reduction of the critical temperature due to spin-wave fluctuations in arsenides and antimonides [37]. Moreover, the reduction is practically canceled by the enhancement of the critical temperature due to hole-hole interactions (see inset of Fig. 1) which explains the success of the mean-field theory in these ferromagnets [37].

\section{Effects of disorder}

Theoretical models beyond the virtual crystal approximation have been used to study the effects of disorder on transport properties, and magnetic properties in 
general, of the DMSs. The Boltzmann equation with the Born approximation scattering rates have provided estimates of the anisotropic magnetoresistance effect (AMR) of order $\approx 1-10 \%$ [38], in good agreement with experiment [11, 13, 34]. AMR of a similar magnitude is predicted by the Kubo formula for the ac-conductivity, as illustrated in Fig. 2. The key for understanding the transport and magnetic anisotropy effects is a strong spin-orbit coupling in the host semiconductor valence band. The absolute dc-conductivity estimates (see the left inset of Fig. 2) [38] are typically only 2-10 times larger than experimental values [11, 12, 39]. The discrepancy is likely partly due to inaccuracies in the model scattering amplitudes and multiple-scattering or localization effects omitted by this approximation. However, experimental conductivities are still increasing as growth and annealing procedures are optimized so the discrepancy could be due mainly to unintended

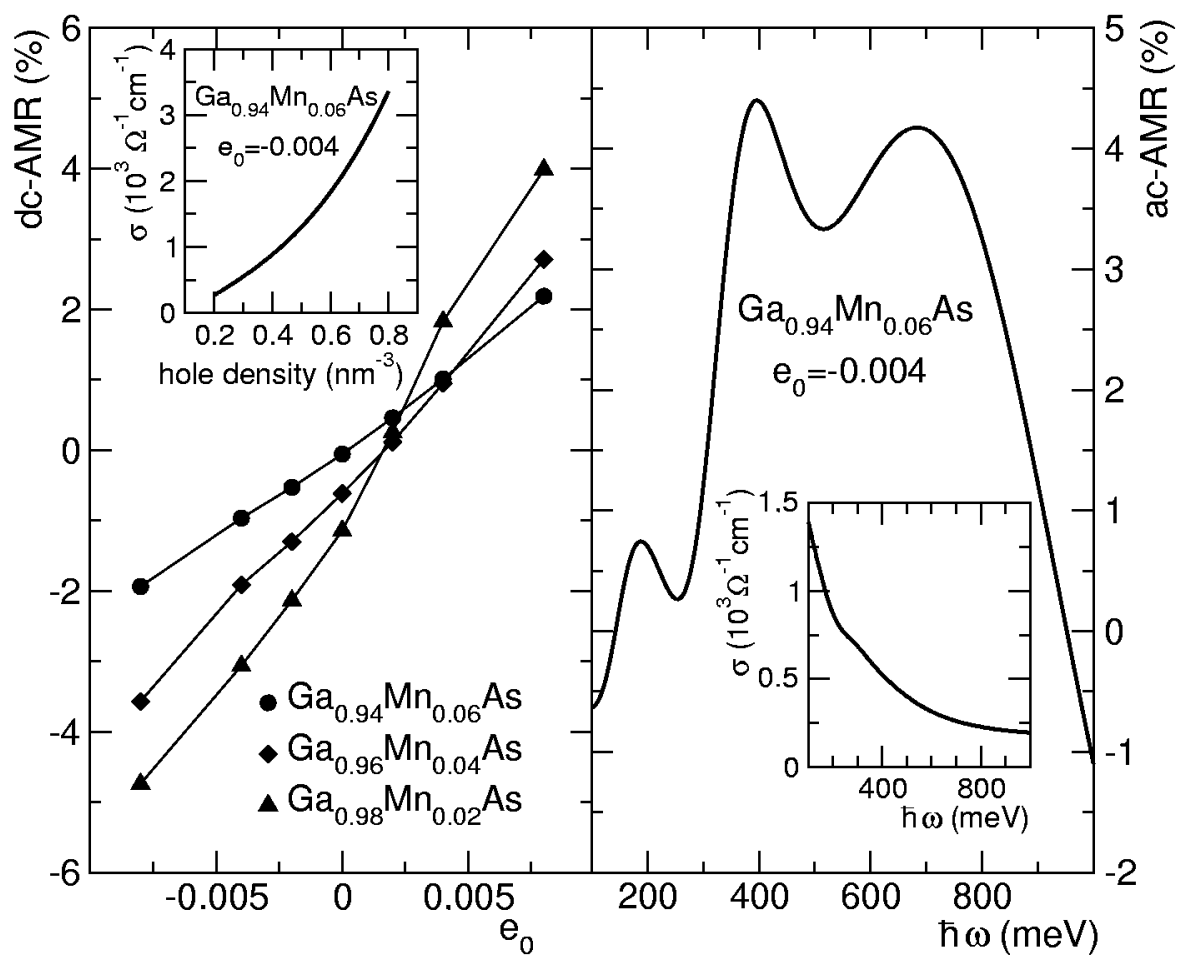

Fig. 2. Left panel: dc anisotropic magnetoresistance as a function of strain for $50 \%$ compensated (due to As-antisites) (Ga,Mn)As DMSs. AMR is defined as the relative difference between resistivities for in-plane magnetization oriented along the current direction and for magnetization perpendicular to the plane of the DMS epilayer. $e_{0}$ is the relative difference between the substrate and the epilayer lattice constants. Inset shows the absolute conductivity for magnetization along the current direction. Right panel: ac-AMR as a function of frequency. Inset shows the absolute ac-conductivity. 
extrinsic disorder. Kubo formula calculations of infrared conductivities, illustrated in the right inset of Fig. 2, of metallic (Ga,Mn)As DMSs demonstrate that transverse $f$-sum rule measurements can be used to extract accurate values for the free-carrier density [40].

The most striking feature in off-diagonal conductivity coefficients of $(\mathrm{Ga}, \mathrm{Mn})$ As and other arsenide and antimonide DMSs is the large anomalous Hall effect (AHE). The AHE occurs because of spin-orbit interactions. In metals [41] the standard assumption has been that the AHE occurs because of a spin-orbit coupling component in the interaction between band quasiparticles and crystal defects, which can lead to skew scattering that give Hall resistivity contributions proportional to $\rho$ and $\rho^{2}$ respectively, where $\rho$ is the diagonal resistivity. Our AHE theory differs fundamentally from this standard picture because the effect arises from spin-orbit coupling in the Hamiltonian of the perfect crystal which implies a finite Hall conductivity even without disorder. In Fig. 3 we plot theoretical anomalous Hall conductivities calculated for a clean system and within a model which accounts for the finite lifetime broadening of the quasiparticle states due to impurity scattering. Figure 3 demonstrates that whether or not disorder is included, the theoretical anomalous Hall conductivities are of order $10 \Omega^{-1} \mathrm{~cm}^{-1}$ in the

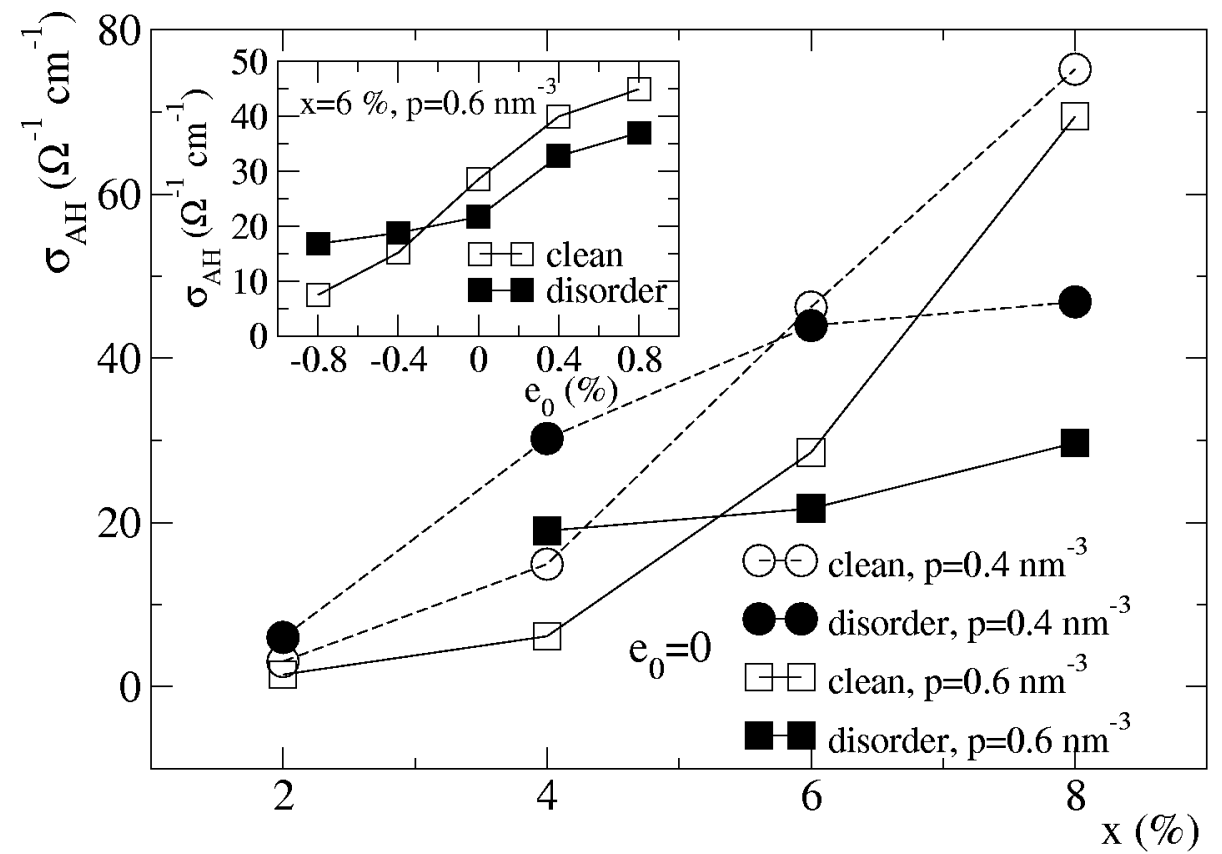

Fig. 3. Theoretical anomalous Hall conductivity of $\mathrm{Mn}_{x} \mathrm{Ga}_{1-x} \mathrm{As}$ DMS calculated in the clean limit (open symbols) and accounting for the random distribution of $\mathrm{Mn}$ and As-antisite impurities (filled symbols). 
(Ga,Mn)As DMSs with typical hole densities, $p \approx 0.5 \mathrm{~nm}^{-1}$, and Mn concentrations of several per cent, consistent with experiment $[1,34]$.

In the infrared regime the theory predicts a prominent feature in the anomalous Hall conductivity at a frequency that varies over the range from 200 to $400 \mathrm{meV}$, depending on the Mn and carrier densities, and is associated with transitions between heavy-hole and light-hole bands. The mid-infrared response feature translates into strong magneto-optical effects, as illustrated in Fig. 4.

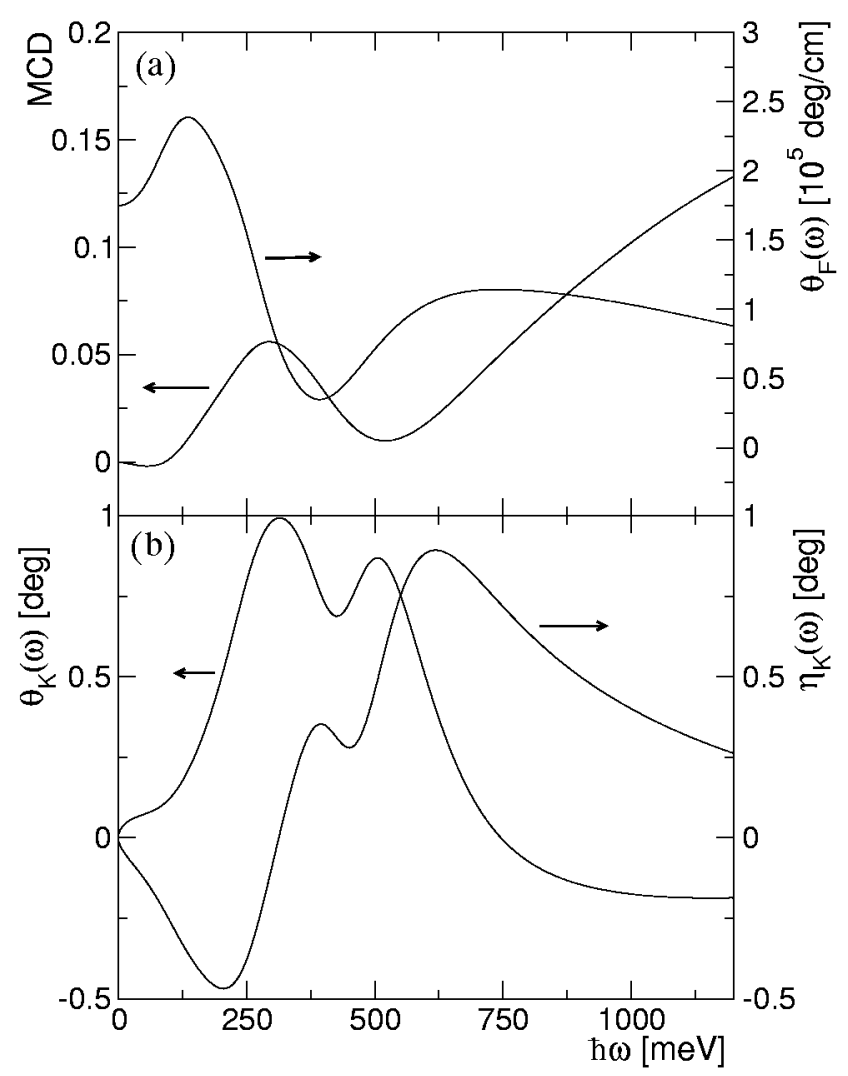

Fig. 4. (a) Faraday and (b) Kerr effects for $x=6 \%$ Mn concentration and $p=$ $0.4 \mathrm{~nm}^{-3}$.

The hole-fluid effective Hamiltonian theory discussed above uses no free parameters and is expected to be reliable in samples with high critical temperatures where the holes are metallic and their interaction with $\mathrm{Mn}$ and other impurities is effectively screened. More detailed studies of disorder effects in DMSs have combined the Kondo description of the spin interactions with Monte Carlo techniques applied to both metallic [42] (high carrier density) and insulating [43] (low carrier density) regimes, and with the CPA [44] or spin-wave theory [45] for the metallic 
samples. The CPA calculations, which start from a tight-binding approximation band model and use the Ruderman-Kittel-Kasuya-Yoshida (RKKY) theory of the effective $\mathrm{Mn}-\mathrm{Mn}$ interaction, predict a factor of 3 enhancement of the critical temperature due to disorder for typical Mn and hole densities in (Ga,Mn)As DMSs [44]. Other studies using dynamical mean field theory [46] suggest similar trends with some additional features when considering the effective $J_{p d}$ coupling as a variable. On the other hand, the random $\mathrm{Mn}$ distribution and spatial fluctuations of the magnetization suppress the ferromagnetic transition temperature in Monte Carlo simulations [42]. The non-collinear nature of the ground state due to disorder has been pointed out in the spin-wave theory calculations [45]. It was also suggested [47] that highly frustrated magnetic correlations may result from the strong spin-orbit interaction in the valence band and the spatial disorder.

The metal-insulator transition regime was explored by exact diagonalization techniques $[48,49]$. Consistent with experiment, the studies predict a metalinsulator transition for 1\% Mn doping in GaAs DMSs [48, 49]. It has been emphasized [48] that defect correlations play an important role in the transition and the calculations $[48,49]$ have provided evidence in favor of the applicability of hole-fluid and impurity-band magnetic-polaron models on metallic and insulating sides of the transition, respectively. Monte Carlo studies of the insulating phase have found spatially inhomogeneous magnetizations below the ferromagnetic critical temperature and an enhancement of the critical temperature due to disorder [43].

\section{Acknowledgments}

We acknowledge helpful discussions with Tomasz Dietl, and Jan Mašek who also provided figures presented in this review. The work was supported by the Grant Agency of the Czech Republic, DARPA, DoE, and the Welch Foundation.

\section{References}

[1] H. Ohno, Science 281, 951 (1998).

[2] T. Dietl, Semicond. Sci. Technol. 17, 377 (2002).

[3] J. Blinowski, P. Kacman, T. Dietl, cond-mat/0201012.

[4] J.M. Langer, C. Delerue, M. Lannoo, H. Heinrich, Phys. Rev. B 38, 7723 (1988).

[5] J. Kreissl, W. Ulrici, M. El-Metoui, A.-M. Vasson, A. Vasson, A. Gavaix, Phys. Rev. B 54, 10508 (1996).

[6] J. Okabayashi, A. Kimura, O. Rader, T. Mizokawa, A. Fujimori, T. Hayashi, M. Tanaka, Phys. Rev. B 58, R4211 (1998).

[7] J. Szczytko, A. Twardowski, M. Palczewska, R. Jablonski, J. Furdyna, H. Munekata, Phys. Rev. B 63, 085315 (2001).

[8] B. Beschoten, P.A. Crowell, I. Malajovich, D.D. Awschalom, Phys. Rev. Lett. 83, 3073 (1999). 
[9] H. Ohldag, V.Solinus, F.U. Hillebrecht, J.B. Goedkoop, M. Finazzi, F. Matsukura, H. Ohno, Appl. Phys. Lett. 76, 2928 (2000).

[10] H. Ohno, J. Magn. Magn. Mater. 200, 110 (1999).

[11] D.V. Baxter, D. Ruzmetov, J. Scherschligt, Y. Sasaki, X. Liu, J.K. Furdyna, C.H. Mielke, Phys. Rev. B 65, 212407 (2002)

[12] K.W. Edmonds, K.Y. Wang, R.P. Campion, A.C. Neumann, N.R.S. Farley, B.L. Gallagher, C.T. Foxon, Appl. Phys. Lett. 81, 4991 (2002).

[13] K.Y. Wang, K.W. Edmonds, R.P. Campion, L.X. Zhao, A.C. Neumann, C.T. Foxon, B.L. Gallagher, P.C. Main, C.H. Marrows, to be published in Proc. 26th Int. Conf. on Physics of Semiconductors, Edinburgh (UK) 2002.

[14] T. Wojtowicz, G. Cywinski, W.L. Lim, X. Liu, M. Dobrowolska, J.K. Furdyna, K.M. Yu, W. Walukiewicz, G.B. Kim, M. Cheon, X. Chen, S.M. Wang, H. Luo, cond-mat/0303212.

[15] H. Akai, Phys. Rev. Lett. 81, 3002 (1998).

[16] M.L. Reed, N.A. El-Masry, H.H. Stadelmaier, M.K. Ritums, M.J. Reed, C.A. Parker, J.C. Roberts, S.M. Bedair, Appl. Phys. Lett. 79, 3473 (2001).

[17] T. Sasaki, S. Sonoda, Y. Yamamoto, K. Suga, S. Shimiz, K. Kindo, H. Hori, J. Appl. Phys. 91, 7911 (2002).

[18] S. Sonoda, H. Hori, Y. Yamamoto, T. Sasaki, M. Sato, S. Shimizu, K. Suga, K. Kindo, cond-mat/0205560.

[19] K. Sato, H. Katayama-Yoshida, Semicond. Sci. Technol. 17, 367 (2002).

[20] S. Sanvito, G. Theurich, N. Hill, J. Supercond. 15, 85 (2002).

[21] J. Mašek, J. Kudrnovský, F. Máca, Phys. Rev. B 67, 153203 (2003).

[22] F. Máca, J. Mašek, Phys. Rev. B 65, 235209 (2002).

[23] S.C. Erwin, A.G. Petukhov, Phys. Rev. Lett. 89, 227201 (2002).

[24] J.H. Park, S.K. Kwon, B.I. Min, Physica B 281/282, 703 (2002); T.C. Schulthess, Bull. Am. Phys. Soc. 2003, March Meeting, K30-1; P.H. Dederichs, K. Sato, H. Katayama-Yoshida, J. Kudrnovský, ibid., S24-5.

[25] J. Okabayashi, A. Kimura, T. Mizokawa, A. Fujimori, T. Hayashi, M. Tanaka, Phys. Rev. B 59, R2486 (1999).

[26] J. König, J. Schliemann, T. Jungwirth, A.H. MacDonald, in: Electronic Structure and Magnetism of Complex Materials, Eds. D.J. Singh, D.A. Papaconstantopoulos, Springer Series in Material Sciences, Vol. 54, Springer, Berlin 2003, p. 163.

[27] Byounghak Lee, T. Jungwirth, A.H. MacDonald, Semicond. Sci. Technol. 17, 393 (2002).

[28] J. Okabayashi, A. Kimura, O. Rader, T. Mizokawa, A. Fujimori, T. Hayashi, M. Tanaka, Phys. Rev. B 64, 125304 (2001).

[29] T. Dietl, H. Ohno, F. Matsukura, Phys. Rev. B 63, 195205 (2001).

[30] M. Abolfath, T. Jungwirth, J. Brum, A.H. MacDonald, Phys. Rev. B 63, 054418 (2001)

[31] T. Dietl, H. Ohno, F. Matsukura, J. Cibert, D. Ferrand, Science 287, 1019 (2000).

[32] T. Jungwirth, W.A. Atkinson, B.H. Lee, A.H. MacDonald, Phys. Rev. B 59, 9818 (1999) 
[33] T. Jungwirth, Q. Niu, A.H. MacDonald, Phys. Rev. Lett. 88, 207208 (2002).

[34] T. Jungwirth, J. Sinova, K.Y. Wang, K.W. Edmonds, R.P. Campion, B.L. Gallagher, C.T. Foxon, Q. Niu, A.H. MacDonald, Appl. Phys. Lett. 83, 320 (2003).

[35] T. Dietl, J. König, A.H. MacDonald, Phys. Rev. B 64, R241201 (2001).

[36] J. König, H.H. Lin, A.H. MacDonald, Phys. Rev. Lett. 84, 5628 (2000).

[37] T. Jungwirth, J. König, J. Sinova, J. Kučera, A.H. MacDonald, Phys. Rev. B 66, 012402 (2002).

[38] T. Jungwirth, M. Abolfath, J. Sinova, J. Kučera, A.H. MacDonald, Appl. Phys. Lett. 81, 4029 (2002).

[39] S.J. Potashnik, K.C. Ku, R. Mahendiran, S.H. Chun, R.F. Wang, N. Samarth, P. Schiffer, Phys. Rev. B 66, 012408 (2002).

[40] J. Sinova, T. Jungwirth, S.-R. Eric Yang, J. Kučera, A.H. MacDonald, Phys. Rev. $B$ 66, 041202 (2002).

[41] C.L. Chien, C.R. Westgate, The Hall Effect and Its Applications, Plenum, New York 1998.

[42] J. Schliemann, J. König, A.H. MacDonald, Phys. Rev. B 64, 165201 (2001).

[43] R.N. Bhatt, M. Berciu, M.P. Kennett, X. Wan, J. Supercond. 15, 71 (2002).

[44] G. Bouzerar, J. Kudrnovský, P. Bruno, cond-mat/0208596.

[45] J. Schliemann, A.H. MacDonald, Phys. Rev. Lett. 88, 137201 (2002); J. Schliemann, cond-mat/0209675.

[46] A. Chattopadhyay, S. Das Sarma, A.J. Millis, Phys. Rev. Lett. 87, 227202 (2001).

[47] G. Zaránd, B. Jankó, Phys. Rev. Lett. 89, 047201 (2002).

[48] C. Timm, F. Schäfer, F. von Oppen, Phys. Rev. Lett. 89, 137201 (2002).

[49] S.-R. Eric Yang, A.H. MacDonald, Phys. Rev. B 67, 155202 (2003). 